\title{
Nucleus-nucleus collisions : what we have learned from the heavy ion program at CERN
}

\author{
A. Capella ${ }^{\mathrm{a} *}$ \\ ${ }^{a}$ Laboratoire de Physique Théorique et Hautes Energies \\ Université Paris XI - bâtiment 211, 91405 Orsay Cedex, France
}

In the first part, I give a brief description of the quark-gluon plasma search at CERN and of some experimental results. In the second part, I review a dynamical model of nucleus-nucleus interactions and propose a physical interpretation of those results.

\section{QUARK-GLUON PLASMA SEARCH}

Heavy ion collisions is a very active field in particle physics. At present the maximal energy is reached at CERN where lead ions are accelerated to an energy of $160 \mathrm{GeV} / \mathrm{c}$ per nucleon. The next machine will be the RHIC collider, expected to operate in 1999, in which gold or lead ions will reach a c. of m. energy of $\sqrt{s}=200 \mathrm{GeV}$. One decade later an energy of $\sqrt{s}=5.5 \mathrm{TeV}$ will be reached at CERN-LHC. The aim of these programs it to produce and detect a new state of matter called quark matter (QM) or quarkgluon plasma (QGP). Since ordinary matter is composed of quark and gluons it is natural to think that by heating and/or compressing it, a new phase of deconfined quarks and gluons can be reached. This idea is quite old and was first explored in the framework of the bag model. However, it only gained full credibility when lattice calculations showed that such a phase transition does occur in statistical QCD.

\subsection{Lattice QCD results}

In the case of a pure Yang-Mills theory (i.e. QCD without quarks) the phase transition is of first order and takes place at a critical temperature $T_{C} \sim 150 \mathrm{MeV}$. In the Big Bang theory the same transition occurred, in the opposite direction (i.e. from QGP to ordinary nuclear matter), when the universe was only a few microseconds

\footnotetext{
*Laboratoire associé au Centre National de la Recherche Scientifique - URA D0063
}

old. Schematically, the situation is as follows. For $T<T_{c}$ one heats the system and the temperature increases whereas the ratio $\varepsilon / T^{4}$, where $\varepsilon$ is the energy density, remains constant. At $T=T_{c}$ one can heat the system but the temperature does not increase (only the latent heat does). Finally, for $T>T_{c}$ the temperature increases again and $\varepsilon / T^{4}$ remains constant. This new constant is much larger than the corresponding one for $T<T_{c}$. This is due to the larger number of degrees of freedom of a QGP as compared to a pion gas. Using the value of the new constant and the value of $T_{c}$ one finds that the phase transition takes place when the density of the system is an order of magnitude larger than that of ordinary nuclear matter. It is also found that there exists a restoration of chiral symmetry which takes place at the same temperature $T_{c}$.

When quarks are introduced, the calculations are more complicated and the order of the phase transition depends on the number of light quark flavors.

\subsection{QGP production}

Although QGP could be produced in the case of neutron stars we are interested here in its production in heavy ion collisions. The first remark is that, in this case, we are a priori very far from the conditions of thermodynamical equilibrium assumed in the lattice calculations in statistical QCD. However, as far as the energy density is concerned, it is plausible that values of the order required by theory (i.e. $1 \mathrm{GeV} / \mathrm{fm}^{3}$ ) can 
be reached in central $\mathrm{Pb}-\mathrm{Pb}$ collisions at present CERN energies. In the Bjorken scenario, the energy density is given by

$\varepsilon=\frac{[d N / d y]_{y^{*} \sim 0}<E_{T}>}{\pi R_{P_{b}}^{2} \tau_{0}}$,

where $d N / d y$ is the number of secondaries per unit rapidity, $\left\langle E_{T}\right\rangle$ is the average transverse energy per particle, and $\tau_{0}$ is the plasma formation time. This time is very poorly known and there is no clear consensus among experts in the field. Using $\tau_{0}=1 \mathrm{fm}$ together with the experimental values of the other quantities in (11), one gets $\varepsilon \sim 2 \div 3 \mathrm{GeV} / \mathrm{fm}^{3}$.

\subsection{QGP signals}

Even if QGP is produced, the phenomenon is a transient one, and one has to find experimental signals of its formation. Although some signals regarding the bulk properties of the production have been proposed in the past (i.e. behavior of $\left.<p_{T}\right\rangle$ versus $d n / d y$ ), it seems at present that the best signals correspond to rear events or to electromagnetic processes. I will only discuss here two of these signals : strangeness enhancement, and $J / \psi$ suppression.

Due to chiral symmetry restoration it is expected that the ratio of strange over non-strange particles will increase due to a reduction of the production threshold in dense hadronic matter.

The suppression of $J / \psi$ production, proposed by Matsui and Satz, is a consequence of color screening in a plasma (Debye screening) - similar to the screening of electric charge in an ordinary plasma. This screening produces a modification of the potential of the type

$V(r)=V_{0}(r) \exp \left(-r / r_{D}(T)\right)$

where $r_{D}$ is the Debye radius - which decreases when $T$ increases. When $r_{D}(T)$ is smaller than the binding radius, the corresponding bound state cannot exist. Of course, at sufficiently high $T$, there will be a transient melting of all hadrons. The interest of the $J / \psi$ (as well as other charmonium and bottonium states) is twofold. First, lattice QCD calculations show that the melting of $J / \psi$ occurs at temperatures $T \sim 1.2 T_{C}$ only slightly above the critical temperature and, second, $c$ and $\bar{c}$ being very rear will combine with light quarks to produce open charmed mesons and therefore, once melted, the $J / \psi$ will not be formed again.

\subsection{Experimental situation}

The two phenomena described above have been observed experimentally in a very clear way. Indeed it has been found that the values of the ratios

$$
\frac{K}{\pi}, \frac{\Lambda}{\pi}, \frac{\bar{\Lambda}}{\pi}, \frac{\Xi}{\Lambda}, \cdots
$$

increase between $p p$ or $p A$ and central $A B$ collisions. The NA35 collaboration claims that there is no increase between $p p$ and $p A$, but errors are still large.

$J / \psi$ suppression has been observed by measuring the ratio $J / \psi$ over Drell-Yan (DY). It is found that this ratio decreases continuously from $p p$ to $p A$ to $A B$ collisions. Furthermore, for a given $A B$ system, the ratio decreases with increasing centrality. This effect is very strong in $P b-P b$ collisions (see Section 2.6 and Fig. 8).

\subsection{Physical interpretation}

There are claims in the literature that strangeness enhancement and particularly the enhancement of multi-strange antibaryons indicates the formation of QGP. Nevertheless, it has also been possible to explain these data in the framework of string models - at the cost of introducing some new ingredients such as string fusion and final state rescattering of secondaries (see section 2.5 ). Likewise, alternative explanations of the observed $J / \psi$ suppression have been proposed (see 2.6).

In the following I will briefly review an independent string model : the dual parton model (DPM) and will discuss to what extent the above signals can be understood in this framework, what are the required modifications of the model and what these heavy ion experiments teach us regarding the mechanism of multiparticle production. 


\section{STRING MODELS AND QGP SI- GNALS}

The dual parton model (DPM) is a dynamical model aimed at describing the mechanism of multiparticle production in hadronic and nuclear collisions. A very similar model, the quark gluon string model (QGSM) has been introduced by the ITEP group. These models are based on the large- $N$ expansion of non-perturbative QCD. A comprehensive review can be found in ref. [1]. A short description of the physical basis of the model and some of its applications to hadronic collisions can be found in [2]. Here I will concentrate on the generalization of the model to nuclear collisions, and, in particular, on the eventual modifications of the model required to explain the CERN heavy ion data.

\subsection{The model}

In $p p$ collisions the main contribution consists in a color exchange producing two strings of type diquark quark (see Fig. 1). The hadronic spectra of each string is obtained by a convolution of the momentum distribution and fragmentation functions of the constituents at the string ends. Due to energy-momentum conservation, the longitudinal momentum fractions of the constituents at the string ends in each hemisphere add up to unity. There are also more complicated configurations, corresponding to higher order terms in the large- $N$ expansion, involving $4,6, \ldots$ etc strings, with sea quarks and antiquarks at their ends. These configurations correspond to multiple inelastic scattering in an $S$-matrix approach. In $p p$ collisions, these higher order configurations give small contributions at moderate energies, but their contribution becomes increasingly important with increasing energy. In proton-nucleus and nucleus-nucleus collisions, these higher-order contributions are enhanced by trivial combinatorial factors (proportional to $A^{1 / 3}$ in $p A$ collisions). More precisely, in $p A$ collisions the dominant graph, involving only one struck nucleon of $A$ and $A-1$ spectator nucleons, is the same two string diagram of Fig. 1. A double inelastic collision, involving two struck nucleons of $A$ and $A-2$ spectators, corresponds to a four string graph as
Figure 1. Dominant two string component in nucleon-nucleon scattering.

shown in Fig. 2, and so on. The weights of these contributions can not be computed in QCD and are taken from the Glauber-Gribov approach.

One assumption of DPM, which is not motivated by the large- $N$ expansion, is that strings are independent and particles emitted in different strings are uncorrelated. This assumption works well in $p p, p A$ and peripheral $A B$ collisions. However, we shall see that for central $A B$ collisions the CERN data require the introduction of final state interaction of secondaries.

Apart from this assumption the model has a sound theoretical basis and has great predictive power. The latter is due to the fact that strings are universal. The momentum distribution and fragmentation functions, allowing to compute the properties of each individual string, are the same in all hadronic and nuclear collisions. Moreover, the momentum distribution functions are determined from Regge intercepts. For instance, that of a valence quark in a pion is given by

$\rho_{q_{v}}^{\pi}\left(x_{q_{v}}\right) \propto x_{q_{v}}^{-1 / 2}\left(1-x_{q_{v}}\right)^{-1 / 2}$.

The behaviour for $x \rightarrow 0$ is the well known $1 / \sqrt{x}$ behaviour associated to an ordinary Regge trajectory of intercept $1 / 2$. Since $x_{q_{v}}+x_{\bar{q}_{v}}=1$, the behaviour for $x_{q_{v}} \rightarrow 1$ is the same as for $x_{\bar{q}_{v}} \rightarrow 0$, and therefore one has the symmetric behaviour (4). In this way the end point behaviour is un- 
Figure 2. Four string component in nucleonnucleus scattering for two inelastic collisions.

der control. Of course, we can multiply (4) by a smooth function of $x$. However, in view of the singular behaviour at the end points, this will have little influence on the numerical results.

For a valence quark in a proton, one has instead

$\rho_{q_{v}}^{p}(x) \propto x^{-1 / 2}(1-x)^{1.5}$.

Here the behaviour at $x \rightarrow 1$ is obtained by taking the diquark momentum fraction to zero. This behaviour is controlled by an exotic $q q \bar{q} \bar{q}$ Regge trajectory of intercept close to $-3 / 2$. As a consequence of (5) the valence quark in a proton is slow in average whereas the diquark is fast $\left(x_{q_{v}}+x_{q q}=1\right)$. Its fragmentation into a leading baryon accounts, in this way, for the leading particle effect (see Section 2.3).

In the first applications of DPM there was no attempt to compute the fragmentation functions. Only their universality was used. For instance in studying $p A$ collisions, one can obtain the fragmentation function from a fit to $p p$ data (or the $p A$ data for a given $A$ ). The model then predicts both the $A$ dependence and the energy dependence. An important progress in the model happened when Kaidalov showed [3] that the fragmentation functions can also be determined to a large extent from Regge arguments. In this way
Figure 3. Feynman $x$ distribution of proton and antiproton in $p p$ collisions at $200 \mathrm{GeV} / \mathrm{c} \mathrm{com-}$ pared with the data of ref. [30] at $175 \mathrm{GeV} / \mathrm{c}$. Diffraction is not included in the calculation.

the model becomes very predictive and the spectra of different hadrons can be computed basically without free parameters - except absolute normalizations.

In order to illustrate this point, I show in Figs. 3 and 4 the leading proton and leading $\Lambda x_{F}$ distribution in $p p$ collisions [4]. The shape of the leading proton spectrum is practically fixed theoretically (here we show only the non-diffractive part but the diffractive one can also be computed in a similar way [5]). Moreover, the different shapes of the proton and $\Lambda$ distributions is entirely due to the difference between $\alpha_{R}(0)=1 / 2$, which appears in the power of $1-x_{F}$ for the leading proton, and $\alpha_{\phi}(0)=0$, which controls the $x_{F} \rightarrow 1$ behaviour of the $\Lambda$-distribution. Likewise, the sharp decrease of $\bar{p}$ and $\bar{\Lambda}$ distributions, also shown in Figs. 3 and 4, is entirely controlled by known Regge intercepts [4], [5].

\subsection{Nucleus-nucleus collisions}

The generalization of the model to nucleusnucleus collisions is rather straightforward. For 
Table 1

The number of charged secondaries per unit rapidity at $y^{*}=0$ for average $\left(\rho_{0}^{\text {aver }}\right)$ and central collision $\left(\rho_{0}^{\text {central }}\right)$ at RHIC and LHC [6].

\begin{tabular}{cccc}
\hline & $\sqrt{s}$ & $\rho_{0}^{\text {aver. }}$ & $\rho_{0}^{\text {central }}$ \\
\hline $\mathrm{SS}$ & $200 \mathrm{GeV}$ & 45 & 166 \\
$\mathrm{SS}$ & $7 \mathrm{TeV}$ & 134 & 503 \\
$\mathrm{~Pb} \mathrm{~Pb}$ & $200 \mathrm{GeV}$ & 513 & 2030 \\
$\mathrm{~Pb} \mathrm{~Pb}$ & $7 \mathrm{TeV}$ & 1890 & 7900 \\
\hline
\end{tabular}

Figure 4. Feynman $x$ distribution of $\Lambda$ and $\bar{\Lambda}$ produced in $p p$ collisions at $200 \mathrm{GeV} / \mathrm{c}$ are compared to a compilation of data from ref. [5].

simplicity I consider here $A A$ collisions. In the approximation of only two strings per nucleonnucleon collision, the rapidity distribution of secondaries is given by

$$
\begin{aligned}
& \frac{d N^{A A}}{d y}(y)=\bar{n}_{A}\left[N^{q q_{A_{P}}-q_{A_{T}}^{v}}(y)+N^{q_{A_{P}}^{v}-q q_{A_{T}}}(y)\right] \\
& +2\left(\bar{n}-\bar{n}_{A}\right) N^{q_{s}-\bar{q}_{s}}(y) .
\end{aligned}
$$

Here $N(y)$ are the rapidity distributions of the individual strings, $\bar{n}_{A}$ is the average number of wounded nucleons of $A$ and $\bar{n}$ is the average number of nucleon-nucleon collisions. Both $\bar{n}_{A}$ and $\bar{n}$ can be computed in the Glauber model. For instance for an average collision (i.e. integrated over impact parameter), one has

$\bar{n}=A^{2} \sigma_{N N} / \sigma_{A B} \propto A^{4 / 3}$.

Note that the total number of strings is $2 \bar{n}$, i.e. two strings per inelastic nucleon-nucleon collision.

The interpretation of (6) is obvious. With $\bar{n}_{A}$ struck nucleons, we have at our disposal $\bar{n}_{A}$ diquarks of projectile and target $\left(q q_{A_{P}}\right.$ and $q q_{A_{T}}$, respectively) and as many valence quarks. This accounts for the first term of (6). The remaining strings : $2\left(\bar{n}-\bar{n}_{A}\right)$ have to be stretched by sea quarks and antiquarks, because the available valence constituents are all included in the first term ; this accounts for the second term of (6). Of course we should combine the valence and sea constituents of the projectile with those of the target in all possible ways. However, for linear quantities such as multiplicities, each ordering gives practically the same result.

We can see from (6) and (7) that, if all strings would have the same plateau height (i.e. the same value of $N(0)$ ), the plateau height in an average $A A$ collision would increase like $A^{4 / 3}$. However, at present energies, the plateau height of the $q_{s^{-}}$ $\bar{q}_{s}$, strings is smaller than that of $q q-q$ ones, and the first term of (5) dominates. At higher energies the contribution of the sea strings becomes increasingly important. Therefore, in order to make predictions for RHIC and LHC we have to introduce the multistring configurations in each nucleon-nucleon collision. If their average number is $2 \bar{K}$ (this number can be computed in a generalized eikonal model; one gets $\bar{K} \simeq 2$ at $\sqrt{s}=200$ and $\bar{K} \approx 3$ at $\sqrt{s}=7 \mathrm{TeV}$ ) the total number of strings is $2 \bar{K} \bar{n}$, and eq. (6) is changed into

$$
\begin{aligned}
& \frac{d N^{A A}}{d y}(y)=\bar{n}_{A}\left[N^{q q_{A_{P}}-q_{A_{T}}^{v}}(y)+N^{q_{A_{P}}^{v}-q q_{A_{T}}}(y)\right. \\
& \left.+(2 \bar{K}-2) N^{q_{s}-\bar{q}_{s}}\right]+\left(\bar{n}-\bar{n}_{A}\right) 2 \bar{K} N^{q_{s}-\bar{q}_{s}} .
\end{aligned}
$$

The predictions $\| 6]$ for average and central $(b \approx$ 0) $S S$ and $P b-P b$ collisions at RHIC and LHC energies are given in Table 1. 
Figure 5. Same as Fig. 2 for the diquark breaking component.

\subsection{Diquark breaking and the leading baryon}

¿From Eq. (5) it follows that the diquark is fast in average and carries a large longitudinal fraction of the incoming proton. Its fragmentation will produce a leading baryon as illustrated in Figs. 1 and 2. These configurations correspond to the fragmentation of the diquark as a whole (the two valence quarks of the diquark find themselves into the same final state baryon), and will be called in what follows diquark preserving (DP) components. However, the diquark can also break in the way illustrated in Figs. 5 and 6, which we shall call in what follows diquark breaking (DB) components. It was stressed in [7] that these two components give contributions to the central plateau height which have different energy behaviour. Therefore when studying $p p$ collisions in a broad range of energies, one should keep track separately of these two components. This is even more necessary in nuclear collisions since the two components have very different $A$ dependence (the ratio of $D B$ over the $D P$ components increases with $A$ ). This is very important since the $D B$ component produces baryons which are slower than those produced by the $D P$ one.

In order to simplify the presentation I discuss
Figure 6. Same as Fig. 5 but here the produced baryon is formed by three sea quarks.

$p A$ collisions. Following [8], I divide the total nucleon-nucleon cross-section into a $D P$ piece and a $D B$ one : $\sigma_{i n}=\sigma_{D P}+\sigma_{D B}$. I assume that once the diquark has been destroyed in a collision with one nucleon of the nucleus it cannot be reconstructed in further collisions with other nucleons of the nucleus. The $N A$ cross-section involving $n$ inelastic $N N$ collisions is then given by

$$
\begin{aligned}
& \sigma_{D B, n}^{N A}(b)=\left(\begin{array}{c}
A \\
n
\end{array}\right) \sum_{i=1}^{n}\left(\begin{array}{c}
n \\
i
\end{array}\right) \sigma_{D B}^{i} \sigma_{D P}^{n-i} T_{A}^{n}(b) \\
& \times\left[1-\sigma_{i n} T_{A}(b)\right]^{A-n} .
\end{aligned}
$$

Here $T_{A}(b)$ is the standard nuclear profile function at impact parameter $b$, normalized to unity. In Eq. (9) we have replaced the usual factor $\sigma_{i n}^{n} T_{A}^{n}$ corresponding to the crosssection for $n$ inelastic collisions by the product $\sum_{i=1}^{n}\left(\begin{array}{c}n \\ i\end{array}\right) \sigma_{D B}^{i} \sigma_{D P}^{n-1} T_{A}^{n}=\left(\sigma_{i n}^{n}-\sigma_{D P}^{n}\right) T_{A}^{n}(b)$. Indeed only the term $\sigma_{D P}^{n} T_{A}^{n}$ will contribute to the diquark preserving cross-section. Summing in $n$ we 
Figure 7. Rapidity distribution of the $p-\bar{p}$ difference in peripheral and central $S S$ collisions (solid curves) [8]. The dashed curves are obtained with $\sigma_{D B}=0$. The data are from [11]. The dotted curve is our prediction for the nucleon minus antinucleon rapidity distribution in central $\mathrm{Pb}-\mathrm{Pb}$ collisions.

have

$\sigma_{D B}^{N A}(b)=\sum_{n=1}^{A} \sigma_{D B, n}^{N A}(b)=1-\left[1-\sigma_{D B} T_{A}(b)\right]^{A}$

Eq. (10) shows that the diquark preserving crosssection belongs to a class of processes [9] which has only self-absorption (or self-shadowing). Obviously

$\sigma_{D P}^{N A}(b)=\sigma_{i n}^{N A}(b)-\sigma_{D B}^{n A}(b)$.

Since $\sigma_{D B}<\sigma_{i n}$, it is clear from Eqs. (10) and (11) that $\sigma_{D B}^{N A}$ increases with $A$ faster than $\sigma_{D P}^{N A}$. Actually, when $\sigma_{D B}$ is sufficiently small to neglect in Eq. (19) second and higher powers of $\sigma_{D B}$, $\sigma_{D B}^{N A}$ will increase linearly with $A$. This proves the result stated above that the relative size of the $D B$ component increases with increasing $A$. The result can be easily generalized to an $A B$ collisions. We have [10]

$\sigma_{D B}^{A B}(b)=1-\left(1-\sigma_{D B} T_{A B}(b)\right)^{A B}$

where $T_{A B}(\mathbf{b})=\int d^{2} s T_{A}(\mathbf{s}) T_{B}(\mathbf{b}-\mathbf{s})$. For $\sigma_{D B}$ sufficiently small we get from (12), after integration in impact parameter, $\sigma_{D B}^{A B}=A B \sigma_{D B}$.

In the numerical calculations, I take $\sigma_{D B}=7$ mb corresponding to a $20 \%$ weight of the $D B$ component. Using eq. (12) one finds that this weight has increased to $40 \%$ for central $S S$ collisions and is as large as $64 \%$ in central $\mathrm{Pb}-\mathrm{Pb}$ collisions.

The results for peripheral and central $S S$ collisions at CERN energies are shown in Fig. 7 and compared with the data from the experiment NA35 11]. We also show the results obtained with $\sigma_{D B}=0$. We see that the dramatic dip present in the latter case for central $S S$ collision has been largely filled in by the contribution of the $D B$ component in agreement with experiment. The prediction for a central $P b-P b$ collision is also shown. In this case the dip is converted into a broad plateau - in agreement with preliminary data from the NA49 collaboration [12].

\subsection{DPM and QGP signals}

In the following I will discuss a possible interpretation of two QGP signals (strangeness enhancement and $J / \psi$ suppression) in the framework of DPM as well as the required modifications of the model - such as string fusion and final state interaction of secondaries. I will also discuss possible consequences of string fusion for cosmic ray physics.

\subsection{Strangeness enhancement}

How can one explain in DPM the enhancement of the ratios (3) ? Since the average number of strings increases with $A$ (or with centrality), and since the extra strings involve sea quarks and antiquarks at their ends, strangeness will be enhanced provided the fraction of $s$-quarks in the sea is larger than the ratio of strange over nonstrange particles at present energies. However, this mechanism is numerically important only for kaons. $\Lambda / \bar{\Lambda}$ production in a $q_{s}-\bar{q}_{s}$ string is negligeable at present energies due to threshold effects. A possible way to enhance $\Lambda / \bar{\Lambda}$ production is via 
string fusion. When two strings overlap, both in transverse space and in rapidity, their momenta as well as their quantum number can merge. If for instance two strings $u \bar{s}$ and $s \bar{d}$ fuse into a $u s-$ $\bar{s} \bar{d}$ one, it will be easy to produce a $\Lambda / \bar{\Lambda}$ pair. In this way it has been possible to explain $\bar{\Lambda}$ enhancement [13].

While this is a very plausible mechanism, the evidence for string fusion is not yet compelling. Indeed, one can assume that diquark-antidiquark pairs are present in the proton sea (with the same relative amount required in the string breaking process in order to produce baryon pairs) [14]. This mechanism is equivalent to string fusion in order to explain $\bar{\Lambda}$ enhancement but does not have some of the consequences of string fusion that will be discussed later.

A limitation of the string fusion mechanism is that it gives the same enhancement of $\Lambda$ and $\bar{\Lambda}$ in absolute value. Experimentally the enhancement of $\Lambda$ is much bigger. What can produce it ? First, the $D B$ component introduced in Section 2.3 is a source of $\Lambda$-enhancement at mid-rapidities. Indeed, in the $D B$ diagram of Fig. 6 the probability to produce a $\Lambda$ is three times larger than in the $D P$ component of Fig. 2 since the strange quark can be any of the three sea quarks that form the baryon. For the $D B$ diagram of Fig. 5 this probability is two times larger than that of the DP component. However, the calculation shows that the obtained $\Lambda$-enhancement is substantially smaller than the experimental one 15. Another source of $\Lambda$ enhancement is therefore needed. Such a source is provided by final state interaction of the produced secondaries. The most important interactions turn out to be 4. 15, 16]

$\pi+N \rightarrow K+\Lambda\left(K^{*}+\Lambda, \cdots\right)$

$\pi+\bar{N} \rightarrow K+\bar{\Lambda}\left(K^{*}+\bar{\Lambda}, \cdots\right)$.

Since the strange particle yield is proportional to the product of densities of the interacting particles, it is clear that $\Lambda$ enhancement will be more important than that of $\bar{\Lambda}$ due to the larger density of nucleons as compared to antinucleons. Moreover, the reactions

$\pi+\Lambda \rightarrow K+N \quad, \quad \pi+\bar{\Lambda} \rightarrow K+\bar{N}$,
Table 2

$\Lambda$ rapidity distribution in central $S S$ collisions at $200 \mathrm{GeV} / \mathrm{c}$ per nucleon computed with and without final state interaction (FSI) compared to data from the NA35 collaboration [17].

\begin{tabular}{cccc}
\hline$y^{*}$ & No FSI & With FSI & NA35 \\
\hline 0 & 0.78 & 1.9 & $2.2 \pm 0.3$ \\
0.5 & 0.77 & 1.8 & $2.1 \pm 0.3$ \\
1 & 0.75 & 1.7 & $2.1 \pm 0.3$ \\
1.5 & 0.70 & 1.6 & $2.2 \pm 0.3$ \\
2 & 0.62 & 1.3 & $1.4 \pm 0.3$ \\
\hline
\end{tabular}

Table 3

$\Lambda$ rapidity distribution in central $P b P b$ collisions at $160 \mathrm{GeV} / \mathrm{c}$ per nucleon computed with and without final state interaction (FSI).

\begin{tabular}{ccc}
\hline$y^{*}$ & No FSI & With FSI \\
\hline 0 & 8.4 & $23 \div 31$ \\
0.5 & 8.1 & $22 \div 30$ \\
1 & 6.8 & $20 \div 25$ \\
1.5 & 5.0 & $16 \div 19$ \\
2 & 2.9 & $8.8 \div 9.1$ \\
\hline
\end{tabular}

which produce a decrease of $\Lambda$ and $\bar{\Lambda}$, are less important than (13)-(14) due to the smaller density of $\Lambda(\bar{\Lambda})$ as compared to nucleons (antinucleons).

Calculations along these lines have been performed in [4, 15, 16. The results for central $S S$ collisions are given in Table 2 and compared with experiment [17]. The agreement is reasonably good. We see that final state interaction is needed in order to reach agreement with experiment. Predictions for central $\mathrm{Pb} \mathrm{Pb}$ collisions are given in Table 3. The value with final state interaction is consistent with preliminary data from the NA49 collaboration [18] (23 \pm 7 at $\left.y^{*} \sim 0\right)$.

\subsection{J/- suppression}

We turn next to $J / \psi$ suppression [19]. For all $p A$ and $A B$ data [20] involving a light projectile (up to $S$ ) there is an alternative explanation to Debye color screening proposed in 19. This explanation is based on the following mechanism [21]. In the present experimental conditions the $J / \psi$ is produced outside (behind) the 
Figure 8. The ratio $B_{\mu \mu} \sigma(J / \psi) / \sigma(D Y)$ versus the interaction length $L$ for $p p, p A, S U$, and $P b$ $P b$ interactions. Data are from refs. [20,22]. The straight line is obtained with nuclear absorption alone. The points labelled theory are obtained [25] with nuclear absorption plus final state interaction of the $J / \psi$ bound state.

nucleus. However, the $c \bar{c}$ pair is produced inside the nucleus and can interact with nucleons in its path through the nucleus. This interaction can modify its wave function in such a way that it has a vanishing projection into the $J / \psi$ bound state. A single absorptive cross-section, $\sigma_{a b s} \approx$ $6.2 \mathrm{mb}$, allows to describe all data except the $\mathrm{Pb} \mathrm{Pb}$ ones. This is illustrated in Fig. 8. The data 22] for $\mathrm{Pb} P b$, also displayed in this figure, show clearly that nuclear absorption alone does not work. Another "conventional" mechanism is the final state interaction of the $J / \psi$ bound state with other secondaries produced at comparable velocity (co-movers) producing open charm :

$\pi+J / \psi \rightarrow D+\bar{D}+\cdots$
This idea was introduced in the literature a long time ago [23]. However, recent theoretical calculations have shown that the $\pi+J / \psi$ and $N+J / \psi$ cross-sections increase very slowly from threshold (in contrast to $\psi^{\prime}+\pi$ which has a very rapid increase) 24]. Therefore in the case of the $J / \psi$ the co-mover cross-section is expected to be very small (not larger than $0.5 \mathrm{mb}$ ). Because of that the idea of $J / \psi$ suppression via interaction with co-movers has been progressively abandoned.

In a recent paper 225] we have shown that introducing the final state interaction (16) with a cross-section of $0.4 \mathrm{mb}$, one can explain the recent $\mathrm{Pb} \mathrm{Pb}$ data. The results are shown in Fig. 8. A similar result has been obtained independently in [26].

One should note, however, that when introducing the interaction with co-movers one has to reduce the value of the cross-section for nuclear absorption. In this way the $J / \psi$ yield in $p A$ collisions does not fall with increasing $A$ as steeply as the data seem to indicate (the "effective" absorptive cross-section resulting from the combined effect of nuclear absorption and interaction with co-movers is $5 \mathrm{mb}$ while experimental data require $6.2 \pm 0.7 \mathrm{mb}$ ). Moreover the $J / \psi$ yield in $S U$ falls somewhat faster than indicated by experiment. At present, however, the data are not precise enough to rule out such an explanation and further study is needed to distinguish it from a QGP scenario [27] and/or other collective effects such as string percolation 28].

There are, of course, many other interesting results obtained at CERN which I have not mentioned. In particular Bose-Einstein correlation measurements (interferometry) indicate that the size of the interaction region is larger than the geometrical size of the colliding system. Again, this requires the existence of final state interaction of secondaries.

\subsection{Consequence of string fusion for cos- mic ray physics}

As discussed in section 2.5, there are hints in the data in favor of string fusion, although there is no compelling evidence yet. Nevertheless, it is interesting to examine its possible consequences in cosmic ray physics. In a recent paper the San- 
Figure 9. Total average multiplicity versus primary energy for a fixed composition assuming string fusion (solid line) and with no string fusion and a composition that changes with energy (dashed line). See text for details.

tiago group [29] discusses two such consequences: the rise of the average shower depth of maximum for cosmic rays in the energy range $10^{16}$ to $10^{19}$ $\mathrm{eV}$ and the possibility that string fusion acts as a hadronic accelerator.

When the energy increases, string fusion becomes more important and produces a decrease of the average multiplicity. Taking a fixed chemical composition (i.e. independent of energy), consisting of $90 \%$ of iron and $10 \%$ of protons, one gets an average multiplicity, which, in the range $10^{17}$ to $10^{19} \mathrm{eV}$, is identical to the one obtained without string fusion and a chemical composition of primaries changing with energy (from $75 \% \mathrm{Fe}$ and $25 \% \mathrm{p}$ at $10^{16} \mathrm{eV}$ to $50 \% \mathrm{Fe}$ and $50 \% \mathrm{p}$ at $10^{19} \mathrm{eV}$ ). This is shown in Fig. 9.

Another interesting consequence of string fusion is that it produces secondaries outside the kinematical range of a nucleon-nucleon interaction. The authors can explain in this way the so-called cumulative effect. Moreover, they claim that these two effects can change the profile of extensive air showers and in this way alter conclusions on the primary composition and energy. For example, provided their composition could be determined by an independent method, cosmic ray events with energies around $3.10^{20} \mathrm{eV}$ would contain, in the presence of string fusion, particles with $\left|x_{F}\right|>2$ or 3 (or more in the presence of string percolation $28 \mid$ ), and would thus correspond to an energy for the primary upto $2 \div 4$ times smaller than the one without string fusion. Such a reduction in energy could make these events compatible with the cut-off due to the scattering of cosmic rays with the microwave background.

\section{CONCLUSIONS}

The QGP search has gained full credibility with the lattice QCD results. Experimental data are of very high quality but give only some hints of QGP formation. Future data and especially future machines will be decisive in this search.

In the mean time, we have gained new insight in the dynamics of multiparticle production allowing to improve current models (strong baryon stopping hinting at diquark breaking, string fusion with interesting consequences for cosmic ray physics and final state interaction of secondaries).

Finally, I want to stress once more that the CERN heavy ion program has produced very many interesting results not mentioned here.

\section{REFERENCES}

1. A. Capella, U. Sukhatme, C.-I. Tan and J. Tran Thanh Van, Phys. Rep. 236 (1994) 225.

2. A. Capella, Proceedings 22nd International Cosmic Ray Conference, Dublin, Ireland, 1991.

3. A. B. Kaidalov, Yad. Fiz. 45 (1987) 1452.

4. A. Capella, A. Kaidalov, A. Kouider Akil, C. Merino and J. Tran Thanh Van, Z. Phys. C70 (1996) 507.

5. A. B. Kaidalov and O. I. Piskunova, Sov. J. Nucl. Phys. 41 (1985) 816.

6. A. Capella, C. Merino and J. Tran Thanh Van, Phys. Lett. B265 (1991) 415. 
7. B. Z. Kopeliovich and B. G. Zakharov, Z. Phys. C43 (1989) 241.

8. A. Capella and B. Z. Kopeliovich, Phys. Lett. B381 (1996) 325.

9. A. Blankenbecler, A. Capella, C. Pajares, A. V. Ramallo and J. Tran Thanh Van, Phys. Lett. B107 (1981) 106.

10. C. Pajares and A. V. Ramallo, Phys. Rev. D31 (1985) 2800.

11. NA35 collaboration, H. Ströbel et al., Nucl. Phys. A525 (1991) 59c.

12. NA49 collaboration in Proceedings XXXI Rencontres de Moriond, Les Arcs (France), 1996 ed. J. Tran Thanh Van ; see also NA44 collaboration, ibid.

13. N. Armesto, M.A. Braun, E. G. Ferreiro and C. Pajares, Phys. Lett. B344 (1995) 301. References to earlier papers on string fusion can be found in G. Gustafson, Nucl. Phys. A566 (1994) 233c. RQMD : H. Sorge, R. Matiello, A. von Keetz, H. Stöcker, W. Greiner, Z. Phys. C47 (1990) 629 ; H. Sorge, M. Berenguer, H. Stöcker, W. Greiner, Phys. Lett. B289 (1992) 6 ; Th. Schönfeld et al., Nucl. Phys. A544 (1992) 439c.

14. J. Ranft, A. Capella, J. Tran Thanh Van, Phys. Lett. B320 (1994) 346 ; H. J. Möhring, J. Ranft, A. Capella, J. Tran Thanh Van, Phys. Rev. D47 (1993) 4146 (the calculations in these papers are based on the DPMJET and DTNUC codes).

15. A. Capella, Phys. Lett. B364 (1995) 175.

16. A. Capella, preprint LPTHE Orsay 96-30, hep-ph 96-05216, Phys. Lett. B in press.

17. NA35 collaboration, T. Alber et al, Z. Phys. C64 (1994) 195.

18. NA49 collaboration, Proceedings QM 96, Heidelberg.

19. T. Matsui and H. Satz, Phys. Lett. B178 (1986) 416.

20. NA38 collaobration, C. Baglin et al., Phys. Lett. B201 (1989) 471 ; Phys. Lett. B255 (1991) 459.

21. R. Salmeron, Nucl. Phys. A566 (1994) 199c. A. Capella, J. A. Casado, C. Pajares, A.V. Ramallo and J. Tran Thanh Van, Phys. Lett. B206 (1988) 354. A. Capella, C. Merino, C. Pajares, A. V. Ramallo and J. Tran Thanh
Van, Phys. Lett. B230 (1989) 149. C. Gerschel and J. Hüfner, Phys. Lett. B207, 253 (1988) ; Z. Phys. C56 (1992) 71.

22. NA50 collaboration, P. Bordaló et al., Proceedings XXXI Rencontres de Moriond 1996 ibid . M. Gonin et al., Proceedings Quark Matter 96, ibid.

23. J.P. Blaizot and J.Y. Ollitrault, Phys. Rev. D39 (1989) 232. S. Gavin, M. Gyulassy and A. Jackson, Phys. Lett. 207 (1988) 194. J. Ftacnik, P. Lichard and J. Pitsut, Phys. Lett. B207 (1988) 194. R. Vogt, M. Parakash, P. Koch and T. H. Hansson, Phys. Lett. B207 (1988) 263. S. Gavin and R. Vogt, Nucl. Phys. B345 (1990) 1104. S. Gavin, H. Satz, R. Thews and R. Vogt, Z. Phys. C61 (1994) 351.

24. G. Bhanot and M. E. Peskin, Nucl. Phys. B156 (1979) 365. A. Kaidalov and P. Volkovitsky, Phys. Rev. Lett. 69 (1992) 3155. A. Kaidalov, Proceedings XXVIII Rencontres de Moriond (1993), ed. J. Tran Thanh Van. M. Luke et al, Phys. Lett. B288 (1992) 355. D. Kharzeev and H. Satz, Phys. Lett. B306 (1994) 155.

25. A. Capella, A. Kaidalov, A. Kouider Akil and C. Gerschel, LPTHE 96-55, hep-ph/9607265.

26. S. Gavin et R. Vogt, Proceedings QM 96, ibid and preprint LBL-37980 (1996).

27. J. P. Blaizot and J. Y. Ollitrault, Proceedings Quark Matter 96, ibid; Phys. Rev. Lett. 77 (1996) 1703. C. Y. Wong, Proceedings Quark Matter 96, ibid. D. Kharzeev and H. Satz, Proceedings QM96, ibid.

28. N. Armesto, M. A. Braun, E. G. Ferreiro and C. Pajares, Phys. Rev. Lett. 77 (1996) 3736.

29. N. Armesto, M. A. Braun, E. G. Ferreiro, C. Pajares and Yu M. Shabelski, preprints USFT/6-96 (Phys. Lett. B to appear) and USFT/11-96 (Astropart. Phys. to appear).

30. A. E. Brenner et al., Phys. Rev. D26 (1982) 1497. 
This figure "fig1-1.png" is available in "png" format from: http://arxiv.org/ps/hep-ph/9611377v1 
This figure "fig2-1.png" is available in "png" format from: http://arxiv.org/ps/hep-ph/9611377v1 